A N N A L E S Annales de Bretagne et des Pays de l'Ouest

Anjou. Maine. Poitou-Charente. Touraine

$115-4 \mid 2008$

Varia

\title{
Sur les « tracks », « trails » et « paths » de John Muir
}

Éric Kocher-Marbœuf

\section{(2) OpenEdition}

Journals

Édition électronique

URL : http://journals.openedition.org/abpo/221

DOI : 10.4000/abpo.221

ISBN : 978-2-7535-1512-3

ISSN : 2108-6443

Éditeur

Presses universitaires de Rennes

Édition imprimée

Date de publication : 31 décembre 2008

Pagination : 111-126

ISBN : 978-2-7535-0808-8

ISSN : 0399-0826

\section{Référence électronique}

Éric Kocher-Marbœuf, «Sur les «tracks », «trails » et " paths » de John Muir », Annales de Bretagne et des Pays de l'Ouest [En ligne], 115-4 | 2008, mis en ligne le 31 décembre 2010, consulté le 02 mai 2019. URL : http://journals.openedition.org/abpo/221 ; DOI : 10.4000/abpo.221 


\title{
Sur les " tracks ", " trails" et " paths » de John Muir ${ }^{1}$
}

\author{
Éric KOCHER-MARBCUF \\ GERHICO-CERHILIM EA 4270 - Université de Poitiers
}

Terre d'élection de la plupart des technologies innovantes qui bouleversent nos habitudes quotidiennes depuis plus d'un demi-siècle, la Californie fait aujourd'hui le pari de la révolution écologique dans des domaines aussi différents que l'énergie, les transports, les biotechnologies et naturellement les technologies de l'information et de la communication. La Silicon Valley et le campus de Stanford University à Palo Alto au sud de San Francisco, le long de la célèbre highway 101, concentrent des milliers de cerveaux venus du monde entier pour relever les défis sans cesse renouvelés d'une économie numérique globale soucieuse du développement durable ${ }^{2}$.

Le modèle économique innovant californien a constamment été relayé par les dirigeants politiques de l'État. La dernière impulsion est venue du gouverneur républicain Arnold Schwartznegger, élu au capitole de Sacramento à la fin de 2003. Ancien acteur hollywoodien, comme Ronald Reagan qui fut l'un de ses prédécesseurs dans les années 1970, le gouverneur Schwartznegger a su faire montre d'un attachement constant pour les questions environnementales et les placer au centre des enjeux politiques de ce début de siècle et de millénaire. En 2004, alors qu'il devait retenir le thème de la pièce " quarter dollar " $(0,25 \$)$ que la Monnaie américaine (United States Mint) avait choisi d'attribuer à l'État de Californie pour commémorer son entrée dans l'Union, Arnold Schwartznegger a choisi le projet du designer Garett Burke représentant John Muir devant le Half Dome du Yosemite survolé par un condor ${ }^{3}$. Quelques mois tard, le gouverneur a annoncé un ambitieux programme écologique pour le

1. Proposition de traduction «Sur les Pistes, chemins escarpés et sentiers de John Muir".

2. FOUCRIER, Annie, COPPOLANI, Antoine, La Californie : périphérie ou laboratoire?, Paris, L'Harmattan, 2004, 299 p.

3. Âgé d'une quarantaine d'années, originaire de Philadelphie, Garett Burke est diplômé de la Pratt Institute School of Art and Design in New York. Il réside depuis 1985 à Los Angeles et a réalisé des projets graphiques pour les grands studios d'Hollywood 
Golden State ${ }^{4}$, fixant entre autres un objectif ambitieux de réduction de $20 \%$ des émissions de gaz à effet de serre à l'horizon $2020^{5}$.

Le choix de John Muir (1838-1914), figure tutélaire du mouvement environnementaliste, pour rappeler aux citoyens le lien étroit devant unir l'économie domestique et les grands principes environnementaux, est aux antipodes du gadget politico-médiatique quand on connaît le montant des investissements consacrés aux secteurs de la nouvelle économie aux États-Unis. Inconnu en France, John Muir est tout naturellement célébré dans sa ville natale de Dunbar, sur la côte orientale de l'Écosse, où il vécut jusqu'au départ de sa famille pour les États-Unis lors de la crise de 1848. La maison familiale des Muir a été transformée en musée. Deux autres lieux de mémoire rappellent qu'il vécut son adolescence dans le Wisconsin. Le domaine agricole des Muir à Fountain Lake, situé à proximité de la ville de Portage dans le comté de Marquette, fut érigé au rang de National historic landmark par le National Park Service et l'autorité environnementale de l'État dès 1957; d'autre part, le musée historique de l'État présente certaines de ses "drôles de machines " réalisées alors qu'il étudiait à l'université de Madison ${ }^{6}$. Toutefois, c'est en Californie, en particulier dans les innombrables sites touristiques de la Sierra Nevada, que le souvenir de John Muir demeure le plus présent. Ses portraits figurent en grand nombre sur les panneaux des tourism exhibits (expositions permanentes des offices de tourisme) et sur les couvertures d'ouvrages d'histoire locale. Il suffit de lancer le moteur de recherche Google, californien il est vrai, pour découvrir rapidement les très riches sites dédiés à Muir, en particulier ceux du Sierra club $^{7}$, du National park service ${ }^{8}$, du John Muir trust écossais ${ }^{9}$, du John Muir birthplace trust ${ }^{10}$. Il est légitime de recourir à cette richesse documentaire en ligne de qualité en la complétant par celle proposée par les universités californiennes ${ }^{11}$.

ainsi que de grandes sociétés comme Microsoft, Sony, Mattel, Disney, etc. La pièce fut officiellement présentée le 31 janvier 2005.

4. La Californie est surnommée le Golden State depuis la ruée vers l'or de 1848, d'où également le nom de Golden Gate servant à dénommer le goulet permettant l'entrée des navires dans la baie de San Francisco.

5. La Californie fut déjà le premier État au monde à rendre obligatoire le pot catalytique pour les véhicules dès 1972, ce qui induisit des recherches sur les motorisations pour les constructeurs automobiles présents sur le marché américain.

6. Le John Muir Memorial Park fut inauguré le 5 mai 1957. Sa création est largement due à l'action de Sylvester "Syl " Adrian of Montello, qui fut tout à la fois inventeur, propriétaire d'un établissement de loisir, environnementaliste et historien local du Wisconsin. Les archives relatives à la création du parc sont conservées par la bibliothèque HoltAtherton de l'université du Pacifique à Stockton (Californie); site [http://library.pacific. $e d u / h a / m u i r / i n d e x . a s p]$. Concernant les inventions de Muir, voir le site [http://www.wisconsinhistory.org/sitesmuseum.asp].

7. [http://www.sierraclub.org/].

8. [http://www.nps.gov/].

9. [http://www.jmt.org/].

10. [http://www.jmbt.org.uk/].

11. On pense particulièrement aux bibliothèques de l'Université de l'État de Californie (Bancroft Library à Berkeley, fonds de San Joaquin, Los Angeles...), de l'Université du 
John Muir doit être considéré comme le chef de file historique du courant de la "préservation " au sein des environnementalistes américains, dont le pendant est constitué par le courant de la " conservation " incarné par Gifford Pinchot. Jusqu'à nos jours, le mouvement environnementaliste américain a été traversé par cette ligne de clivage entre les deux versants de la protection de la nature qui peut aussi se traduire en un rapport dialectique entre biocentrisme et anthropocentrisme utilitariste ${ }^{12}$. Pour John Muir et ses successeurs, il convient de préserver des sanctuaires naturels vierges de toute influence anthropique, alors que Gifford Pinchot et ses émules prônent une exploitation rationnelle des ressources et la constitution de réserves stratégiques de matières premières avec l'achèvement de la Frontière ${ }^{13}$. Il n'est donc pas étonnant que la réflexion sur les rapports entre l'homme et l'environnement se soit épanouie le long de la côte du Pacifique, dans ces grands espaces du nord-ouest du continent américain qui s'étendent du désert du Nouveau Mexique aux montagnes glacées d'Alaska ${ }^{14}$.

John Muir, quant à lui, n'a jamais théorisé ses actions. Monsieur Jourdain des voyages au long cours, il ne s'est jamais pensé comme un explorateur, mais plutôt comme un randonneur des grands espaces préfigurant l'outdoorsman de l'Amérique actuelle, toujours prêt à affronter les pires conditions physiques ou atmosphériques pour retrouver l'esprit des pathfinders, des pionniers ${ }^{15}$. Notons toutefois que les randonneurs d'aujourd'hui disposent de vêtements et de chaussures en matériaux hightech qui faisaient cruellement défaut à un John Muir qui escaladait des sommets de plus de 3000 mètres et des glaciers en chaussures de ville et veste de tweed! Rien qu'à considérer l'aspect physique si particulier de John Muir, il est évident qu'une personnalité originale, excentrique, pouvant faire penser à celle d'un illuminé, émane de ses traits. En vieillissant,

Pacifique (Holt Atherton Library qui détient les archives de Muir, [http://library.pacific. edu/ha/muir/index.asp]), à la Green library de Stanford University, [http://www-sul.stanford.edu/].

12. DUBAN, François, L'écologisme aux États-Unis : histoire et aspects contemporains de l'environnementalisme américain, Paris, L'Harmattan, 2000, p. 9-10; ERHLICH, Gretel, « John Muir's nature visionary ", National geographic, 2000; Fox, Stephen, John Muir and His Legacy: The American Conservation Movement, Little, Brown and co, 1981.

13. Gifford Pinchot (1865-1946), ancien étudiant de Yale, fut formé en France à l'École nationale des eaux et forêts de Nancy et fut le premier directeur de l'United States Forest Service puis il fut gouverneur républicain progressiste de Pennsylvanie dans les années 1920 et 1930. Voir : MeYER, John M., "Gifford Pinchot, John Muir, and the Boundaries of Politics in American Thought ", Polity, n 30, p. 267-284; MiLler, Char, Gifford Pinchot and the Making of Modern Environmentalism, Island Press, 2001; SмIтн, Michael B., "The Value of a Tree: Public Debates of John Muir and Gifford Pinchot ", The Historian, n ${ }^{\circ} 60$, p. $757-778$.

14. CAIGNOL, Florent, La Nature et l'Homme : Introduction à l'histoire de l'environnement et étude du Nord-Ouest Pacifique (deuxième moitié du XIXe siècle), Nice, Université de NiceSophia-Antipolis, 2005.

15. Un $4 \times 4$ du constructeur automobile japonais Nissan porte ce nom pour séduire la clientèle américaine en faisant référence à la mythologie des grands espaces. 
Muir prend l'allure d'un patriarche, d'un prophète de l'Ancien Testament, peut-être même d'un druide qui aurait revêtu un costume du XIX siècle.

Analyser la portée historique des multiples écrits littéraires et scientifiques de Muir ainsi que leur implication dans les développements du mouvement environnementaliste américain tout au long du $\mathrm{xx}^{\mathrm{e}}$ siècle constitue une plongée dans un tel océan archivistique et documentaire qu'aucun chercheur ne s'y est risqué jusqu'à aujourd'hui. Le sujet réclame de mâ̂triser des connaissances scientifiques variées puisque John Muir est tour à tour présenté comme l'un des grands botanistes, naturalistes, géologues de son temps et surtout comme le plus grand défenseur de la wilderness ${ }^{16}$ américaine ${ }^{17}$. Tous les voyages de Muir ne revêtent pas un intérêt scientifique, certains appartiennent davantage au mythe américain de la quête de la frontière, de la prise de possession du continent vierge à travers ses propres limites physiques. En revanche, tous ces voyages s'inscrivent dans le cheminement d'une existence qui s'est progressivement fixée pour but de chérir et de défendre la nature primitive. À un moment donné, cette quête personnelle a rencontré une partie des préoccupations de la société américaine, précisément au moment où la frontière du Pacifique a été atteinte et qu'une forme de vertige a saisi le pays.

\section{Des brumes de Dunbar aux forêts du Wisconsin Comment s'est forgé un caractère d'exception (1838-1863)}

John Muir naît dans le Lothian, à Dunbar sur la côte écossaise, non loin d'Edimbourg, le 21 avril 1838. Il est le premier fils de Daniel et d'Ann Gilrye Muir qui ont déjà deux filles. L'aînée, Margaret, est née en 1834, sa cadette, Sarah, en 1836. Un second fils, David, naîtra en 1840, suivi encore de jumelles Mary et Annie en 1846 et enfin de Joanna en 1850.

John fréquente l'école élémentaire dès l'âge de 3 ans et entre à la Grammar School de Dunbar à 7 ans. La pédagogie y est stricte et inclut les châtiments corporels. Du matin au soir, le jeune Muir apprend les bases de l'anglais littéraire, du latin, du français, des mathématiques et de la géographie. L'acquisition précoce de ces enseignements fondamentaux lui sera précieuse lorsqu'il se lancera dans les études supérieures en autodidacte. Durant ses trop rares moments de liberté, John erre sur la côte et dans la campagne environnante, seul ou en compagnie de son frère David. Il se passionne pour la faune américaine décrite par Alexander Wilson ${ }^{18}$ et John James Audubon ${ }^{19}$.

\footnotetext{
16. Littéralement la nature à l'état sauvage ou primitif.

17. Une approche globale du personnage a été proposée en 2006 par le John Muir environmental Center de l'Université du Pacifique à Stockton (CA).

18. Alexander Wilson (1766-1813) naît en Écosse mais est le pionnier de l'ornithologie américaine en publiant les huit volumes d'American ornithology entre 1808 et 1814 .

19. John James Audubon (1785-1851), the American woodsman, d'origine française, surpasse Wilson comme ornithologue dans ses 435 planches peintes à la main de Birds of America, publié à Édimbourg en 1826.
} 
En février 1849, Daniel Muir et ses deux fils quittent Dunbar pour le nouveau monde en attendant de faire venir les femmes de la famille. La crise économique sévit en Europe et même si John Muir ne le dit pas explicitement dans ses Souvenirs d'enfance et de jeunesse, c'est sans doute la menace de la misère qui force le père de famille à quitter les ingrates terres d'Écosse ${ }^{20}$. Via le chemin de fer entre Édimbourg et Glasgow, les trois hommes embarquent sur un voilier rempli d'émigrants pour la traversée transatlantique. Comme des millions d'immigrants, ils débarquent à New York. Daniel Muir a d'abord l'idée de s'établir au Canada mais il bifurque à Buffalo pour le comté de Marquette dans le Wisconsin, à Fountain Lake, où la terre est plus facile à travailler, même s'il faut d'abord vaincre la forêt primitive $^{21}$.

John mène la dure vie de fermier jusqu'à ses 21 ans. Il racontera la rudesse de cette existence dans ses Souvenirs d'enfance et de jeunesse (The story of my boyhood and youth) à la fin de sa vie en 1913. Ce livre constitue un témoignage précieux sur les conditions de vie des pionniers et la mentalité de l'Amérique profonde, individualiste, travailleuse, austère, religieuse et hostile aux Indiens ${ }^{22}$. Durant toutes ces années, John ne fréquente plus les bancs de l'école mais apprend par lui-même les mathématiques, la géométrie, la littérature et la philosophie durant l'adolescence. Il se passionne également en cachette pour la littérature et la poésie ainsi que pour les inventions les plus diverses : horloges, baromètres, thermomètres, hygromètres. Cette volonté d'apprendre est d'autant plus méritoire qu'elle heurte les sentiments religieux de Daniel Muir qui estime qu'il n'y a nulle autre connaissance utile que les Saintes Écritures et le travail physique pour servir Dieu. John s'astreint à ne dormir que quelques heures par nuit, de 20 heures à 3 heures du matin, pour pouvoir se cultiver et travailler à ses inventions.

En 1856, lorsque la famille s'établit à Hickory Hill dans une nouvelle ferme, il a 18 ans. Durant la construction, John échappe de peu à la mort par asphyxie en creusant un puits.

En septembre 1860, sous la pression du voisinage qui l'encourage et insiste auprès de son père, John quitte la ferme familiale pour faire connaître ses inventions à l'exposition de Madison; c'est son premier voyage depuis son arrivée dans le Wisconsin onze ans plus tôt! Il est primé et suscite autant d'admiration que d'interrogations chez les notables. En février 1861, après avoir occupé divers emplois, il vainc sa timidité en s'inscrivant à l'université du Wisconsin, à Madison, qu'il fréquente durant deux

20. MuIR, John, Souvenirs d'enfance et de jeunesse (trad. André Fayot), Paris, José Corti, 2004 , p. 40.

21. Ibidem, p. 43.

22. L'attitude de John Muir vis-à-vis des born native American évolue avec l'âge. De franchement hostile, Muir passe à une réelle empathie lorsqu'il découvre les tribus d'Alaska. Cette évolution a donné lieu à une analyse par MERChANT, Carolyn, "Shades of Darkness: Race and Environmental History ", Environmental History, volume 8, n 3, July 2003. 
ans et demi. Afin de financer ses études, il se fait maître d'école en hiver. Le professeur de géologie Ezra Carr, dont John connaît l'épouse prénommée Jeanne depuis l'Exposition, l'initie à la géologie. Il invente un pupitre de travail automatique qui stupéfie autant les étudiants que les professeurs pour son ingéniosité. Grâce à une minuterie, le pupitre se charge d'ouvrir les livres des différentes matières à la page étudiée en fonction des heures programmées! Cette minutie n'est pas un cas isolé aux États-Unis, où la notion de "valeur-temps " existe dès le XIX ${ }^{\mathrm{e}}$ siècle comme en atteste la diffusion des montres ou des horloges et le proverbe time is money. Auditeur libre non rattaché à un cursus académique, John Muir choisit ses matières et se passionne ainsi pour la botanique.

Au printemps 1863, il quitte l'université pour retourner à la Hickory Hill où il pense recevoir sa convocation sous les drapeaux, la guerre de Sécession faisant rage depuis déjà deux ans.

\section{L'errance d'un jeune homme qui cherche à donner un sens à sa vie (1863-68)}

Ne trouvant aucun ordre de route au domicile familial, John Muir se lance dans un premier voyage pédestre à but botanique le long de la rivière Wisconsin jusqu'au Mississippi. En 1864, il s'établit pour deux ans en Ontario, à Meaford, dans la baie Géorgienne du lac Huron. Après divers emplois, il cogère une pêcherie de truites sauvages. Pendant ses moments de loisir, il mène des recherches botaniques et découvre une orchidée la Calypso borealis qui constitue sa première contribution scientifique. Le dimanche, plutôt que d'instruire ses élèves de l'histoire biblique, il préfère leur faire découvrir la botanique. Il entame une correspondance avec Jeanne Carr, l'épouse de son professeur de Madison, qui restera sa vie durant sa confidente et sans doute son grand amour platonique.

Au même moment, en 1864, le président Abraham Lincoln signe un bill donnant la vallée du Yosemite et le Mariposa big tree grove à la Californie en tant que terres de parc d'État. C'est la première pierre de la protection de la wilderness dans la Sierra Nevada et Muir n'a rien à voir avec cette décision.

La guerre de Sécession finie, John Muir revient aux États-Unis en 1866 après la destruction par le feu de l'usine de fumage de truites. Lorsqu'il deviendra célèbre, certains affirmeront que Muir s'est soustrait à la conscription durant la guerre civile, il niera toujours cette version des faits, expliquant qu'il n'a jamais reçu de convocation à la ferme parentale ${ }^{23}$. Une chose est sûre, il ne s'est pas porté volontaire pour se battre dans les rangs des Fédérés. Il devient contremaître puis ingénieur dans une entreprise de construction de véhicules hippomobiles d'Indianapolis, inventant

23. C'est la thèse soutenue entre autres par Roderick NASH, Phd de l'Université de Madison, Wisconsin, professeur d'histoire durant trente ans à l'Université de Californie à Santa Barbara et spécialiste de l'histoire du Wilderness. 
des machines pour automatiser une partie de la production. Fin 1866, le Boston Recorder publie son article sur la Calypso borealis. Le 5 mars 1867, il est gravement accidenté à un œil alors qu'il travaille sur une machine. Il demeure entre la vie et la mort pendant plusieurs jours, et aveugle pendant de longues semaines tout en restant alité dans une chambre noire. Ayant la chance de recouvrer la vue, il décide de quitter l'usine, la vie ordinaire, et choisit d'aller étudier la nature.

Le $1^{\text {er }}$ septembre 1867, John Muir débute sa marche d'un millier de miles. Il veut simplement profiter de la vie dont il a mesuré la fragilité. Il critique l'utilitarisme social et le matérialisme de la société américaine et désire faire le plein de la vue de la nature, se remplir d'elle. Il semble avoir la forêt tropicale d'Amérique du Sud comme but ultime mais à son départ, à Indianapolis, il n'a pas une idée arrêtée du tracé de son périple. Il écrit sur la première page de son journal de voyage « John Muir, planète terre, univers ". John Muir suit une route passant par l'Indiana, le Kentucky, le Tennessee, la Caroline du Nord, la Géorgie et arrive en Floride. Il suit les traces d'autres découvreurs comme Daniel Boone (1732-1820) dans le Kentucky qui fut aussi un pionnier du wilderness avant de céder à la spéculation foncière. Il découvre ses premières vraies montagnes avec les Appalaches et mesure les ravages économiques et démographiques causés par la guerre civile en Caroline du nord où il manque d'être détroussé, voire assassiné par une bande de bandits de grands chemins. À ce moment, il met ses pas dans ceux d'illustres découvreurs tels Hernando de Soto (1500-1542) ${ }^{24}$ et le naturaliste William Bartram (1739-1823) qui passa quatre années entre 1773 et 1877 à parcourir 2400 miles $^{25}$ à travers les futurs États des Carolines, de Géorgie, Tennessee, Alabama, Mississippi et de Floride ${ }^{26}$.

Parvenu à Savanah, Muir embarque jusqu'à Ferdinandina puis gagne Gainesville et Cedar Key sur le golfe du Mexique. Atteint de la malaria, il ne peut embarquer pour Galveston au Texas comme il l'a d'abord envisagé. Il s'embarque pour Cuba où il passe plusieurs semaines à s'extasier sur les beautés de la flore. Retournant à New York depuis La Havane, il décide de repartir par mer pour la Californie à bord d'un paquebot bondé de pionniers décidés à tenter leur chance sur la côte ouest.

\section{La rencontre fusionnelle avec l'Ouest (1868-1879)}

John Muir arrive le 28 mars 1868 à San Francisco où il se sent mal à l'aise, ce qui le décide à suivre un compagnon de traversée se rendant pour ses affaires dans l'intérieur des terres. C'est ainsi qu'il chemine jusqu'à la vallée du Yosemite au printemps 1868. La beauté spectaculaire des lieux provoque chez lui une telle émotion qu'il note dans son carnet qu' " aucun temple bâti de la main des hommes ne peut se comparer avec le Yosemite

24. [http:/nps.gov/deso/].

25. 3860 kilomètres.

26. [http:/bartramtrail.org/]. 
qui est le plus majestueux de tous les temples de la nature ". L'année suivante, en 1869, Pat Delaney, un riche rancher lui propose d'encadrer son berger, dans les pâturages d'estive de ses 4000 moutons. John Muir passe ainsi son "premier été dans la Sierra " dans les Tuolumme Meadows. Il peut s'adonner à l'escalade, à la connaissance des ranges et des glaciers. Il effectue la première ascension du Cathedral Peak (Pic Cathédrale) et du Mont Dana, se penche du haut de la vertigineuse chute du Yosemite et emprunte l'ancestral indian trail entre les Bloody Canyon et le Lac Mono à la beauté minérale hors du commun.

Afin de rester à proximité du Yosemite, Muir entre au service de l'exploitant forestier James Hutchings en 1870. Il s'installe dans une cabane à côté d'une scierie hydraulique qu'il bâtit sur la Merced River. Parallèlement, il entame une activité de guide pour les premiers savants en visite au Yosemite que lui envoie son mentor Jeanne Carr. C'est ainsi que Joseph Leconte (1823-1901), professeur de géologie et de sciences naturelles à l'Université de Californie et le philosophe transcendantaliste Ralph Waldo Emerson (1803-1882) découvrent le site en compagnie de Muir en 1870-1871.

Au fil de ses excursions, Muir forge une théorie inédite de la formation des vallées de la Sierra, expliquant qu'elles sont d'anciennes auges glaciaires et que leur morphologie spectaculaire résulte de l'action mécanique de la glace sur la roche. Il publie ses premières conclusions dans un article intitulé les " glaciers de Californie " dans le New York Tribune du 5 décembre 1871. Il s'agit d'un véritable défi lancé à Josiah Whitney, l'éminent responsable de la mission géologique de Californie, qui prétend que la vallée du Yosemite résulte d'un tremblement de terre de grande magnitude. Qualifié d' " amateur " et même d' " ignorant " par Whitney, Muir est défendu par le célèbre professeur de Harvard Louis Agassiz (1807-1873), lui-même auteur d'une étude sur les âges glaciaires, qui salue en Muir " le premier homme qui ait eu une conception cohérente de l'action glaciaire ». À l'automne 1871, Muir reconnaît la Hetch Valley (ou Tuolumne Yosemite) et un petit cirque glaciaire actif sous le pic Merced qui achèvent de le convaincre de l'origine glaciaire de la morphologie des vallées de la Sierra. Toujours grâce à Jeanne Carr, Muir fait la connaissance de John Tyndall (1820-1888) qui visite les États-Unis en 1872. Ce dernier a été l'un des premiers à étudier les âges glaciaires dans les Alpes; il confirme également les observations de Muir. Enfin, Muir accueille également au Yosemite le botaniste John Torrey (1796-1873), membre de l'Académie américaine des Sciences et président de l'American association for the advancement of science et le jeune Clinton Hart Merriam (1855-1942) sera par la suite le premier directeur de la Division d'ornithologie et des mammifères du département de l'Agriculture, ancêtre du National Wildlife Research Center (NWRC).

1872 est en quelque sorte l'année fondatrice pour la politique de protection de l'environnement aux États-Unis puisque c'est à cette date que 
le Yellowstone est érigé en parc national. C'est aussi une année d'intense activité pour John Muir qui publie une série d'articles dans The Overland Monthly (Yosemite valley in flood, Twenty Hill Hollow, Living Glaciers of Californie). Il reçoit également la visite d'Asa Gray (1810-1888), éminent élève de Torrey, devenu professeur de botanique à Harvard, membre de l'Académie des Sciences et qui succède en 1872 à son illustre maître à la tête de l'American association for the advancement of science. C'est également à ce moment que Muir se lie d'amitié avec le peintre William Keith (1838-1911). Durant l'été 1872, Muir effectue l'ascension du mont Ritter (13000 pieds ${ }^{27}$ ) par la face nord. La renommée de Muir ne cesse de grandir les années suivantes. En 1873, il publie dans le Weekly Transcript de Boston The Hetch hetchy Valley, gravit seul et sans équipement le mont Whitney (14500 pieds ${ }^{28}$ ), point culminant des États-Unis, par une voie inédite et opère une première reconnaissance au Kings River Canyon. En mars 1874, Muir s'attaque en solitaire au mont Shasta dans la chaîne des Cascades (14 162 pieds $^{29}$ ) et explore les lits de lave de Modoc à la frontière entre la Californie et l'Oregon ${ }^{30}$. The Overland Monthly de San Francisco commence la publication de ses Études sur la Sierra. Jeanne Carr, non contente de veiller sur sa renommée grandissante, se met en quête de lui trouver une épouse! C'est ainsi que Muir est bientôt présenté à la jeune Louie Wanda Strentzel, issue d'une famille d'origine polonaise ayant fait fortune dans la culture arboricole à Martinez, non loin d'Oakland, où Muir s'est récemment établi. Les Carr se sont aussi rapprochés de John Muir puisque le professeur Ezra Carr est devenu le premier professeur d'agriculture de la jeune Université de Berkeley en 1873 et l'initiateur de la première ferme-école de l'État, à Davis non loin de Sacramento ${ }^{31}$. Ezra Carr prône rapidement une orientation moins élitiste de l'enseignement que certains de ses collègues et parvient à se faire élire au poste de superintendant des écoles de l'État de Californie en 1875, ce qui lui permet de demeurer membre des trustees de l'université alors qu'il allait être désavoué et contraint à la démission par ses pairs. C'est naturellement par son entremise que John Muir est présenté à John Swetts ${ }^{32}$, lui-même ancien superintendant, très introduit dans l'administration de l'État californien ${ }^{33}$.

Au fil des ans, John Muir poursuit inlassablement ses expéditions scientifiques et sa stratégie de conquête de l'opinion. En 1875, on le retrouve à

27. 3690 mètres.

28. 4418 mètres.

29. 4315 mètres.

30. C'est en ce lieu qu'une cinquantaine de guerriers Indiens commandés par le chef Captain Jack tinrent tête durant cinq mois à des forces dix fois supérieures en nombre de l'US Army lors de la pacification de la région.

31. L'Université de Californie à Davis a attribué le nom de John Muir à son institut sur l'environnement durable, [http://johnmuir.ucdavis.edu/].

32. On trouve aussi l'orthographe John Sweet.

33. Le poste de superintendant existe toujours, il est à cheval entre les fonctions d'inspecteur d'académie et de ministre à l'enseignement scolaire de l'État. John Sweet a été superintendant entre 1863 et 1867, Ezra S. Carr de 1875 à 1880. 
dos de mule pendant trois mois dans la partie méridionale de la Sierra à la découverte des big redwoods, ces fameux séquoias millénaires. Grâce à John Swetts, il commence à s'impliquer dans le débat public californien pour la protection de la forêt et de l'environnement en 1876. Il prononce alors sa première conférence publique à l'Institut littéraire de Sacramento et le Sacramento Record Union publie son vibrant appel au gouvernement pour la protection des forêts millénaires qu'il compare, à la manière de Chateaubriand dans le Génie du christianisme, aux God's first temples, aux premiers temples de la Création. En 1877, Muir conduit Asa Gray et Sir Joseph Dalton Hooker (1817-1911) dans la région du mont Shasta. Hooker est alors le conservateur des jardins royaux de Kew dans le Surrey et est considéré comme le plus grand botaniste britannique du XIX ${ }^{\mathrm{e}}$ siècle. Muir guide également l'expédition de l'US geodetic dans les montagnes du Nevada et de l'Utah et achève ses recherches annuelles en explorant les montagnes de San Gabriel en Californie méridionale.

\section{Les voyages en Alaska (1879-1881) ${ }^{34}$}

En 1879, à 41 ans, John Muir se fiance à Louie Wanda Strentzel qu'il connaît depuis déjà quatre ans, puis il retourne dans la chaîne des Cascades en compagnie de l'alpiniste Renfrew. Dès son retour, Muir se joint à l'expédition du pasteur presbytérien S. Hall Young en partance pour une mission d'évangélisation en Alaska méridional. Lors des préparatifs de l'expédition, Muir fait la connaissance de l'armateur Robert Moran qui lèguera ses terres de l'île Orcas dans l'État de Washington afin qu'elles deviennent un parc d'État en 1921. C'est au cours de ce premier voyage que Muir découvre Glacier bay et Muir Glacier qu'il appelle sur le moment Geike and Hugh Miller glaciers. Après son retour, Muir donne une série de conférences sur ses découvertes à Portland en Oregon, et fréquente les pionniers de l'exploration de la zone du nordouest Pacifique qui reste encore largement inconnue mais où fourmillent les chercheurs d'or. La ruée vers le Klondike va bientôt battre son plein.

Le 14 avril 1880, John Muir se marie avec Louie Wanda Strentzel mais il repart dès juillet pour un deuxième séjour en Alaska. Le mariage ne change guère les habitudes de Muir qui a obtenu le consentement de son épouse pour un " célibat de travail " annuel de plusieurs mois. Ce modus vivendi a contribué à nourrir une légende noire sur le personnage, qui lui prête aussi des relations intimes avec Elvira Hutchings, l'épouse de son ancien employeur, et naturellement avec son mentor Jeanne Carr. C'est au cours de ce deuxième voyage qu'il manque de se perdre dans les crevasses du grand glacier en compagnie d'un chien nommé Stickeen qui sera aussi le titre du livre qu'il tirera de cette aventure. Muir retourne encore en Alaska en 1881, alors même que naît sa fille Wanda Muir ${ }^{35}$.

34. MurR, John, Voyages en Alaska, Paris, Payot, 1995, 344 p.

35. Sa seconde fille, Helen naquit en 1886. 
Sur les " tracks ", " trails " et " paths " de John Muir

\section{De la tentation du repli intimiste au combat pour l'immortalité}

En 1882, ses beaux-parents font bâtir la superbe Martinez mansion devenue propriété du service des parcs nationaux. Muir écrit pour le Century Magazine un article publié en deux volets et intitulé "Bee-Pastures of California " qui deviendra Our national Parks. Il s'installe comme rancher et arboriculteur et le restera pendant huit ans, se révélant excellent gestionnaire. En 1885, il rend visite à sa famille demeurée dans le Wisconsin car il pressent la mort prochaine de son père. Il en profite pour visiter Yellowstone, passe par les gorges de la Columbia et les chutes de Multnomah (620 pieds, $189 \mathrm{~m}$ ). Il attend 1886 pour emmener Louie à Yosemite. Au printemps 1888, bien qu'âgé de 50 ans, John Muir gravit le mont Rainier (14410 pieds, 3490 m) en participant à l'expédition d'Edward Ingraham, qui baptise le camp de base de son nom car Muir a découvert un abri naturel au vent en observant une pierre ponce. Dans la foulée, Muir, décidément infatigable, enchaîne les visites des principaux sites naturels de l'Oregon (Snoqualmie Falls, Spokane Falls, Crater Lake). Les observations qu'il en tire lui permettent d'enrichir les deux volumes de Pictuaresque California entamée l'année précédente et d'écrire Steep trails ("Chemins escarpés ").

Déjà partiellement sorti de sa retraite scientifique avec ses récents voyages et travaux, John Muir est pressé de s'impliquer dans la sauvegarde du Yosemite par Robert Underwood Johnson du Century en 1889. Le directeur du magazine, qui a largement contribué à diffuser les idées de Muir, est alors engagé avec son confrère George W. Steward du Visalia Delta contre la récente décision du General Land Office (GLO) de mettre en vente des lots de forêt comprenant de grands séquoias dans le sud de la Sierra. Natif de Californie, Steward avait réussi depuis le début de la décennie 1880 à sensibiliser l'opinion et les responsables politiques du sud de l'État en faveur de la préservation des arbres et des prairies de la haute Sierra contre les ravages causés par les bûcherons et le pacage intensif des moutons. La nouvelle administration fédérale consécutive à l'élection du républicain Benjamin Harrison en 1888 opère un revirement en faveur des intérêts des exploitants forestiers qui inquiète les défenseurs de la nature primitive ${ }^{36}$. John Muir ne peut demeurer sourd à cet appel. À l'issue de son quatrième voyage en Alaska en 1890, Muir publie deux articles fondamentaux qui lancent la campagne de préservation du Yosemite : "Les trésors du Yosemite " et "Les caractères du parc national du Yosemite ". Muir s'engage aux côtés de Steward pour la création du Sequoia and Kings Canyon National Park, qui ne verra le jour qu'un demi-siècle plus tard. Dès 1891, Muir se rend dans la Kings River.

Comprenant, l'âpreté du combat à mener contre les intérêts économiques, Muir cherche à fédérer les énergies et les soutiens. En 1892, il est à

36. DisAlver, Lary, TweEd, William, Challenge of the big trees, Sequoia natural history association, 1990, chapter IV, "Parks and forests : protection begins " (1885-1916). 
l'origine de la fondation du Sierra club, dont il restera le président jusqu'à sa mort, qui demeure toujours la plus grande puissante organisation de défense de l'environnement aux États-Unis. John Muir est désormais complètement dégagé de la gestion du ranch et libre de revenir dans l'arène publique.

\section{Les voyages de la consécration. Nouveaux horizons (1893-1911)}

Durant la dernière partie de son existence, John Muir ne cesse de voyager, il fait même preuve d'une véritable boulimie de découvertes géographiques, comme s'il voulait contempler les plus beaux sites naturels des cinq continents et découvrir les hauts lieux de culture de l'humanité avant de mourir. En 1893, il se rend sur la côte est, à New York et à Concord dans le Massachusetts où il fleurit les tombes des deux maîtres de la littérature romantique américaine ayant exalté la beauté sauvage de la nature, Henry David Thoreau (1817-1862) et Ralf Waldo Emerson (1803-1882). Ayant franchi l'Atlantique, il retourne sur les lieux de son enfance écossaise à Dunbar et séjourne à Édimbourg puis Londres. Il se rend aussi en Irlande, en Norvège, en Suisse, en France et en Italie pour y découvrir montagnes, fjords et musées.

Les Montagnes de Californie (The Mountains of California), premier " vrai » livre de Muir, paraît en 1894. Le lancement de l'ouvrage s'accompagne d'une campagne en faveur de la création de nouveaux parcs nationaux et d'un retour du Yosemite dans le giron de l'État fédéral. Cette implication aux frontières de la politique, tant la question est débattue par l'opinion, les médias et les décideurs, est suivie deux ans plus tard de la nomination de Muir à la US Forestry Commission qui mène de grandes enquêtes sur la préservation de nombreuses forêts et parcs ${ }^{37}$. Muir se mobilise aussi pour la création d'un parc national du Mont Rainier qui verra le jour en 1899. Son appartenance à l'US forestry commission l'amène à visiter la Roan Mountain dans les Appalaches, située à cheval entre le Tennessee et la Caroline du nord et la Grandfather mountain, point culminant du Blue ridge mountain range en Caroline du nord. Il poursuit vers Montréal, le Saint-Laurent, les montagnes du Maine et du Vermont.

Muir se préoccupe toujours autant des richesses de l'Alaska, il y retourne au sein de l'expédition du paléontologue Henry Fairfield Osborn (1857-1935) en 1896 et l'année suivante dans les Canadian Rockies. Il y effectue un septième voyage en 1899 au sein de l'expédition du magnat du rail Harriman à Wrangell, Glacier Bay, Stitka et dans le détroit du Prince William.

À l'orée du vingtième siècle, l'œuvre de John Muir connaît une véritable consécration. Il reçoit des diplômes d'honneur des universités de Harvard

37. La commission travaille ainsi sur le Yellowstone, les Black Hills, les États de l'Idaho, de l'Oregon, de Washington, la chaîne des Cascades, les coast ranges de Santa Lucia, les montagnes de Californie méridionale, le Grand Canyon et la Sierra Nevada méridionale... 
et du Wisconsin ${ }^{38}$, écrit sur la préservation forestière dans des revues à plus fort lectorat comme le Harper's Weekly, l'Atlantic Monthly, effectue un grand retour au Yosemite et dans la Sierra Nevada en compagnie de C. Hart Merriam en 1900. En 1901, il fait paraître Our national parks et conduit une centaine de membres du Sierra club dans la région du Yosemite pendant un mois. Enfin, en 1903, le président Theodore Roosevelt passe trois jours en compagnie de Muir dans la vallée du Yosemite.

Edward Henry Harriman, le richissime propriétaire de la Southern Pacific railway, offre à Muir de l'accompagner dans son tour du monde. À 65 ans, Muir ne supporte pas l'avalanche des collections des musées de Londres, Paris et Berlin. Il arrive épuisé à Moscou mais se rend néanmoins dans le Caucase puis en Finlande. Grâce au Transsibérien, il rejoint la Mandchourie et de là gagne la Corée, le Japon, la Chine, l'Inde, l'Egypte, Ceylan, l'Australie, la Nouvelle Zélande, l'Indonésie, les Philippines, Hong Kong, Hawaï et finalement San Francisco! En Nouvelle-Zélande, il n'hésite pas à parcourir le glacier Mueller sur le Mont Cook. Ce tour du monde mériterait à lui seul un livre.

En 1905, Muir voyage en Arizona avec sa fille Helen atteinte de problèmes respiratoires, il participe à la rencontre conjointe organisée par les Mazamas et le Sierra club sur les monts Hood et Rainier et va étudier la forêt pétrifiée qui deviendra un monument national en 1906. Il n'est pas là lorsque son épouse Louie décède le 6 août à Martinez. Finalement, Theodore Roosevelt décide le retour de la gestion et de la protection de la vallée du Yosemite à l'administration fédérale à compter de 1906; c'est la grande victoire de Muir qui tempère son chagrin. Ce succès va toutefois être assombri par la bataille pour la sauvegarde de la Hetch Hetchy Valley (Tuolomne Yosemite) que les autorités de San Francisco veulent ennoyer. Les politiques de la Bay Area veulent créer un grand réservoir capable de produire de l'énergie hydroélectrique et de prémunir contre les ravages de nouveaux incendies tels que ceux que la ville a connus en 1906 après le tremblement de terre.

En 1908, le grand Canyon national monument est créé, de même que le Muir woods national monument au nord du Golden gate ${ }^{39}$. Il s'agit d'un " monument naturel ", une forêt de séquoias géants possédée par un millionnaire, William Kent, qui est ainsi sauvée. Cette même année 1908, Muir dicte son autobiographie à Edward Henry Harriman, à Pelican Bay dans l'Oregon, sur le lac Klamath. En 1909, Muir guide le président Taft dans la Sierra Nevada et rencontre le grand ornithologue John Burroughs (18371921). Promeneur inlassable, il accompagne encore des amis en $1910 \mathrm{au}$ Yosemite et dans le grand Canyon.

À plus de 70 ans, Muir prend conscience qu'il serait temps de mettre en forme la montagne de notes qu'il a prises lors de ses randonnées au

38. Il reçut le diplôme d'honneur de Yale en 1912 et fut fait docteur en droit honoris causa par l'Université de Californie en 1913.

39. [http://www.nps.gov/muwo/ et http://www.visitmuirwoods.com/]. 
Yosemite. Il va lui falloir une année pour écrire My first summer in the Sierra qui poétise son expérience initiatique de 1869. Il ne s'agit pas d'un long monologue scientifique et abscons, mais plutôt d'une ode au Yosemite, à la faune et à la flore des montagnes californiennes. Cette rédaction est entrecoupée d'un voyage sur la côte est au printemps 1911. Muir se rend à New York, Washington, Garrison (Hudson Highlands), Boston, New Haven. Il va voir Harriman à Rochester en mai, accorde un entretien au Boston Herald avant une conférence devant les membres de l'Appalachian Mountain Club, il fera de même en juin devant ceux de l'American Alpine club de New York.

\section{Dernier voyage, d'autres Yosemite, l'universalité du message de Muir (1911-1912)}

En août 1911, Muir quitte New York pour un grand voyage vers l'Amérique du Sud et l'Afrique, concrétisant son rêve, vieux de quarante ans, de découvrir les forêts de l'Amazone et du Chili. Depuis Manaus, il découvre la forêt amazonienne en septembre. En novembre, il est au Chili afin de voir les Araucana (Araucaria ou monkey-puzzle tree) et fait une boucle dans le sud des Andes. Il arrive au Cap en Afrique du Sud le 13 janvier 1912 et se rend aux chutes Victoria où il peut admirer les grands baobabs. Il remonte ensuite l'Afrique de l'est et retourne aux États-Unis via le canal de Suez et Naples. Il arrive à New York le 27 mars après avoir effectué un voyage de 40000 miles $^{40}$, il a tout juste 74 ans!

À peine rentré en Californie, il reprend la lutte contre la destruction de la wilderness en s'attaquant aux pratiques des bûcherons, des mineurs et des barons de l'énergie hydroélectrique qui veulent toujours ennoyer la Hetch Hetchy Valley. Cette ultime bataille sera en définitive perdue en 1913. Muir participe au camp d'été 1912 du Sierra Club dirigé par Stephen Mather, futur premier directeur du Service des parcs nationaux. À la rentrée, paraît son livre Yosemite qui sera suivi Story of my boyhood and youth en 1913.

En mai 1914, Muir reçoit, dans son ranch de Martinez, un jeune étudiant japonais du Tacoma Washington's college qui a dévoré ses livres, il s'agit de Ryozo Azuma (1879-1980), pionnier de la défense de l'environnement au pays du soleil levant et surnommé le " John Muir du Japon ". Outre qu'elle rappelle les liens étroits qui existaient déjà entre le Japon et la côte pacifique des États-Unis, cette rencontre revêt une portée prémonitoire si on la met en perspective avec les enjeux environnementaux majeurs soulevés par le protocole de Kyoto et l'essor économique sans précédent de l'Asie.

En décembre 1914, John Muir contracte une sévère pneumonie dans le froid glacial du désert Mojave alors qu'il séjourne chez sa fille Helen à Dagett. Transféré à l'hôpital de Los Angeles, il y décède dans la nuit de Noël 1914. La mort de Muir n'interrompt pas la publication de ses œuvres

40. 64300 kilomètres. 
en préparation. C'est ainsi que les Voyages en Alaska ${ }^{41}$ sont publiés en 1915, La marche d'un millier de miles vers le Golfe (A thousand mile walk to the Gulf) en 1916, La croisière du Corwin (The cruise of the Corwin) en 1917, Chemins escarpés (Steep trails) en 1918. Très vite, le destin de Muir suscite des travaux d'érudition ou de vulgarisation. En 1924, William Badé écrit la Vie et lettres de John Muir. Une biographie de John Muir, Le fils de la Nature (The son of the wilderness) est récompensée par le prix Pulitzer 1945 attribué à Linnie Marsh Wolf.

Plusieurs raisons peuvent expliquer la postérité de John Muir. De nombreux Américains nourrissent envers lui l'admiration traditionnelle qu'ils ont pour les self made men, avec cette triple dimension de force physique, d'intelligence pratique et instinctive qui sied aux vrais héros du nouveau monde. Ces qualités auraient pu lui offrir la fortune mais il a su dépasser l'utilitarisme social et le matérialisme de la mentalité du Golden State. En Alaska, il suscite une ruée vers l'or de milliers d'aventuriers en mentionnant la présence de pépites dans un creek à deux orpailleurs qu'il rencontre au hasard d'une piste. Surtout, John Muir fait comprendre à la société américaine de son temps que la fin de la frontière à l'ouest et la domestication de la dernière nature vierge risquent de briser l'élan vital de la mentalité américaine $^{42}$. Si l'Amérique veut rester elle-même, elle doit préserver une part de cette nature originelle pour s'y ressourcer et la transmettre aux générations futures; l'harmonie de l'homme et de son environnement pourront susciter alors de nouvelles richesses. Muir a donc pressenti la logique d'un développement soucieux de ne pas marquer la planète pour transmettre la biodiversité aux générations futures. Il est fascinant de penser que l'expérience de terrain et les écrits d'un homme aussi particulier que John Muir ont contribué à la pose de l'une des premières pierres de ce mouvement désormais irréversible. En ayant voyagé sur les cinq continents, à la découverte des sites et des espèces animales et végétales les plus emblématiques de la terre, John Muir pressentait déjà que la préservation du village planétaire allait être le défi des générations futures et qu'il s'agissait d'une responsabilité collective de l'humanité tout entière ${ }^{43}$.

\footnotetext{
41. Une édition japonaise fut même publiée en 1942, année du lancement d'un Liberty Ship portant le nom de Muir et destiné à la bataille du Pacifique.

42. Sur les aspects artistiques de la question, voir le catalogue d'une exposition organisée par les musées des Beaux-Arts de Rouen, Rennes et Marseille en 2008. SALOMÉ, Laurent (dir.), La mythologie de l'Ouest dans l'art américain 1830-1940, Milan, Silvana editoriale, 2007, 215 p.

43. Worster, Donald, "John Muir and the Modern Passion for Nature ", Environmental History, January 2005, 10(1), p. 8-19.
} 


\section{Orientation bibliographique}

TURNER, Frederick, Rediscovering America, John Muir in his time and ours, Viking Presse, 1985

TURNER, Frederick, John Muir, New York, Perseus, 2000, 417 p.

MUIR, John, Eight wilderness discovery books, Mountaineers, 1992.

Muir, John, Un été dans la Sierra, Paris, Hoëbeke, 1997, 231 p.

Mur, John, Voyages en Alaska, Paris, Payot, 1995, 339 p.

Mur, John, Souvenirs d'enfance et de jeunesse, Paris, Corti, 2004, 208 p.

MuIR, John, Quinze cents kilomètres à pied à travers l'Amérique, Paris, Corti, 2006, $165 \mathrm{p}$.

MuIR, John, Journal de voyage dans l'Arctique, Paris, Corti, 2008, 296 p. (traduction de The cruise of the Corwin).

Bernard Fillaudeau et Fabienne Raphoz-Fillaudeau, éditeurs de la maison Corti, ont entrepris de faire découvrir l'illustre environnementaliste aux lecteurs de langue française et se sont lancés sur ses traces en parcourant à leur tour certains de ses itinéraires.

\section{RÉSUMÉ}

À l'heure où l'environnement et le développement deviennent un nouveau domaine de recherche pour les historiens à l'échelle mondiale, il apparaît judicieux de s'intéresser à John Muir (1838-1914), figure pionnière de la préservation de la nature sauvage américaine, en particulier celle de la Californie et de la façade du Pacifique. Cet Écossais d'origine, qui passa son adolescence dans le Wisconsin, synthétise bien des aspects originaux de la culture américaine de son époque. Tout à la fois inventeur de machines géniales, explorateur, botaniste, géologue, scientifique, vulgarisateur et même homme d'affaires avisé dans le secteur des agrumes, Muir est parvenu à sauver la vallée du Yosemite de la spéculation foncière. Il est le fondateur du Sierra club, la plus importante association de défense de l'environnement américaine, et son œuvre sert aujourd'hui autant à illustrer des programmes universitaires qu'à servir de symbole pour l'ambitieuse politique environnementale voulue par le gouverneur Arnold Schwartznegger qui a choisi Muir pour figurer sur la pièce de 25 cents de 2005 .

\section{ABSTRACT}

At the time, environment and sustainable development are surely a new scientific field for historians at a world-scale level. In this way, it seems to be interesting to consider John Muir's (1838-1914) life, because he was a pioneer to preserve wilderness especially in California and the Pacific Coast of America as well. Born in Scotland, Muir emigrated to Wisconsin with his parents, studied in Madison and therefore synthesized the new American culture. Genius inventor of mechanic machines, exploratory, botanist, geologist, scientific, popularizer, shepherd, business man in citrus fruits, Muir is above all known to be the Yosemite Valley's protector against land speculators. Sierra Club's founder, his association is still the largest one for environment preservation in the US. His name and work are very often used for academic programmes in universities and he has been chosen by governor Schwartznegger to symbolize California's commitment in environment topics with the quarter dollar in 2005. 